\title{
Anion Bridges Drive Salting Out of a Simple Amphiphile from Aqueous Solution
}

\author{
D. T. Bowron ${ }^{1,2}$ and J. L. Finney ${ }^{2}$ \\ ${ }^{1}$ ISIS Division, Rutherford Appleton Laboratory, Chilton, Didcot, Oxon OX11 0QX, United Kingdom \\ ${ }^{2}$ Department of Physics and Astronomy, University College London, Gower Street, London WC1E 6BT, United Kingdom
}

(Received 5 July 2002; published 1 November 2002)

\begin{abstract}
Neutron diffraction with isotope substitution has been used to determine the structural changes that occur on the addition of a simple salting-out agent to a dilute aqueous alcohol solution. The striking results obtained demonstrate a relatively simple process occurs in which interamphiphile anionic salt bridges are formed between the polar groups of the alcohol molecules. These ion bridges drive an increase in the exposure of the alcohol molecule nonpolar surface to the solvent water and hence point the way to their eventual salting out by the hydrophobic effect.
\end{abstract}

DOI: 10.1103/PhysRevLett.89.215508

Ever since the early studies of Hofmeister [1] the effect of salts on the solubility of amphiphilic molecules has been of intense interest. Despite its regular use for the concentration and crystallization of biological molecules such as proteins, the phenomenon is not well understood. The "classical view"" (see, e.g., [2]) explains the effect in terms of competition between the salt and the protein for water of solvation. Arakawa and Timasheff's experiments [3] indicate that proteins are preferentially hydrated in the presence of salting-out agents, while Chick and Martin [4] suggest that the salt is excluded from the protein precipitate. These latter views argue that the saltingout agents do not interact with the protein and hence question the classical view. Recent NMR studies have argued in favor of direct salt-nonpolar group interactions $[5,6]$, while other views favor an indirect interaction mediated by the hydration shells of the ions and the nonpolar solutes [7].

Recent advances in the application of isotope substitution neutron scattering measurements have given us detailed insight into the structures of aqueous twocomponent amphiphile systems, showing us how amphiphiles interact in aqueous solution as temperature and concentration are varied [8-11]. We present here a significant extension of these techniques to an aqueous solution of tertiary butanol to which a salting-out agent has been added.

To extract the structural correlations in this system a series of isotopically labeled solutions were prepared without and with sodium chloride, a known and simple salting-out agent. The stoichiometric ratio of alcohol to water for all solutions was 1:50, corresponding to a mole fraction concentration of $\approx 0.02$. This concentration is close to the minimum in the relative partial molar volume of $t$-butanol (TBA) in $t$-butanol-water mixtures [12], a region that is traditionally associated with the maximum in hydrophobic effects observed in these solutions [13]. In the solutions containing $\mathrm{NaCl}$, the $\mathrm{NaCl}$ :water ratio was fixed at 1:100, so that the concentration of ions (irrespective of type) is then equal to that of the alcohol molecules.
PACS numbers: 61.20.Qg, 61.12.-q, 64.75.+g, 82.30.Nr

To allow extraction of information relating to the hydration of the alcohol molecules and the structure of the water solvent, five isotopic mixtures were prepared for each of the salt and no-salt cases, namely, (1) $\left(\mathrm{CD}_{3}\right)_{3} \mathrm{COH}$ in $\mathrm{H}_{2} \mathrm{O}$; (2) a $1: 1$ mixture of $\left(\mathrm{CD}_{3}\right)_{3} \mathrm{COH}:\left(\mathrm{CD}_{3}\right)_{3} \mathrm{COD}$ in 1:1 $\mathrm{H}_{2} \mathrm{O}: \mathrm{D}_{2} \mathrm{O}$; (3) $\left(\mathrm{CD}_{3}\right)_{3} \mathrm{COD}$ in $\mathrm{D}_{2} \mathrm{O}$; (4) a $1: 1$ mixture of $\left(\mathrm{CH}_{3}\right)_{3} \mathrm{COH}:\left(\mathrm{CD}_{3}\right)_{3} \mathrm{COD}$ in $1: 1 \mathrm{H}_{2} \mathrm{O}: \mathrm{D}_{2} \mathrm{O}$; (5) $\left(\mathrm{CH}_{3}\right)_{3} \mathrm{COH}$ in $\mathrm{H}_{2} \mathrm{O}$. Using the second order difference neutron scattering technique (see, e.g., [8]), samples (1)(3) give access to the structural correlations in the solutions weighted towards the water molecules, while (3) -(5) target the structural correlations weighted towards both those between the water molecules and those between the alcohol and water molecules.

Neutron scattering measurements were taken on the SANDALS diffractometer at the ISIS pulsed spallation neutron source, U.K. The samples were contained in flat $\mathrm{Ti}_{0.68} \mathrm{Zr}_{0.32}$ alloy cells, with a wall thickness of $1.1 \mathrm{~mm}$ and internal dimensions $1 \mathrm{~mm} \times 35 \mathrm{~mm} \times 35 \mathrm{~mm}$. This alloy composition gives zero coherent scattering contribution to the measured diffraction signal. The samples were maintained at the ambient temperature of the instrument $\sim 25^{\circ} \mathrm{C}$. Corrections were applied for absorption and multiple scattering, and the data normalized to the incoherent scattering of vanadium using the ATLAS suite of programs [14]. Throughout the analysis the atomic densities of all solutions were taken to be 0.1 atom $\AA^{-3}$ (cf. [15]). For the system with added salt, the same measurements were repeated. No isotopic substitutions were made on either the cation or the anion.

In order to examine the structures implied by the experimental data, the empirical potential structure refinement technique (EPSR) was used $[16,17]$. This generates model assemblies of molecules that are consistent with the experimental diffraction data and has been used extensively in earlier studies of aqueous amphiphile systems $[8,9,11]$. These assemblies can then be interrogated and a range of structural quantities extracted. These include partial radial distribution functions (rdfs) as well as functions giving information on molecular orientations 
[18]. EPSR was implemented with model cubic boxes of side length $\approx 21.5 \AA$, containing 300 water molecules and six alcohol molecules. For the salt-containing system, three $\mathrm{Na}^{+}$and three $\mathrm{Cl}^{-}$ions were included.

To establish a structural reference we consider first the salt-free system, specifically the interactions that occur between solute molecules and between solute and water molecules. The results are found to be fully consistent with earlier work on a $0.06 \mathrm{~mol}$ fraction aqueous $t$-butanol solution [9]. The solute-solute interaction is dominated by contact between the nonpolar head groups (the three methyl groups), as indicated by the cap above the nonpolar head group in the spatial density function (SDF) plotted in Fig. 1(a). As noted earlier [8], this approach geometry is exactly as would be expected for a classical hydrophobically driven interaction. Coordination numbers (CC-CC, Table I) show that each $t$-butanol molecule is in contact with on average 1.5(0.7) neighbors in this interaction. As indicated from the alcohol oxygen-water oxygen coordination number, the polar tail of the amphiphile interacts on average with 2.5(0.6) water molecules; again as noted earlier [9], the alcohol oxygen-alcohol oxygen coordination numbers in Table I show that there is a very low probability indeed of direct association of two solute molecules through direct hydrogen bonding. Other solvent molecules "girdle" the equatorial nonpolar region of the $t$-butanol at a distance of approximately $5 \AA$, as seen in the SDF of Fig. 1(b). This figure also shows that the water molecules around the polar tail (i.e., beneath the $t$-butanol molecule) are more closely associated with the
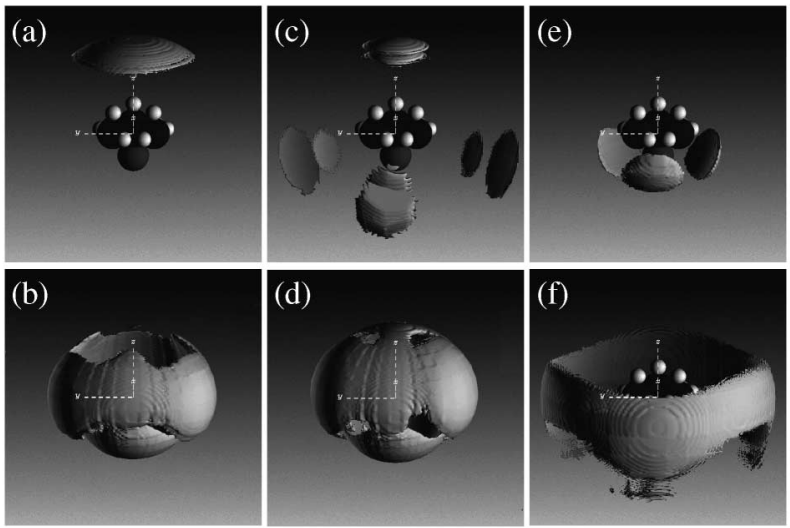

FIG. 1. Spatial distribution functions of various components of the solutions surrounding the $t$-butanol molecules. Through the orientational placing of the lobes, these plots show how particular neighboring molecules are distributed around a typical central $t$-butanol molecule. (a) and (c) $t$-butanols around a central $t$-butanol, (a) salt-free solution, (c) including salt; (b) and (d): water surrounding a central $t$-butanol, (b) salt-free solution, (d) including salt; (e) and (f): ions surrounding a central $t$-butanol molecule, (e) chloride, (f) sodium. The viewport for each panel is set to $16 \AA$ by $16 \AA$ with the alcohol molecule at the center. central alcohol molecule than are the equatorial waters. This is a direct result of their hydrogen bonding to the alcohol hydroxyl group and results in a distance between the central carbon atom (CC) of the alcohol molecule and a water oxygen of approximately $3.7 \AA$.

We now compare the results obtained with added $\mathrm{NaCl}$. First, with respect to the solute association, the effect of adding salt is dramatic. Consider first the rdf between the central carbon atoms of neighboring $t$-butanol molecules (Fig. 2). The first peak centered close to $6 \AA$ corresponds to the head-to-head contacts of the salt-free case (solid line) - the molecules in the "cap" of Fig. 1(a). Upon adding salt (dashed line), this peak is much reduced, and an additional peak appears, centered on a distance of between $8-8.5 \AA$. Integrating under these peaks shows that (Table I) between about one-third and one-half of the nonpolar associated molecules have been displaced, with an approximately equivalent number appearing at the longer distance. The question then arises as to the chemical nature of this more distant interaction and how this relates to the addition of salt (cation and/or anion) to the system.

This central question can be answered by referring to the TBA-TBA SDF of the added-salt system shown in Fig. 1(c). The original capping lobe of Fig. 1(a) - which indicated nonpolar-nonpolar head-to-head interactionshas been reduced, as expected from the reduced first peak in Fig. 2. Consistent with this reduction, there is a corresponding increase in the solvent density in the head group cap region, as illustrated in Fig. 1(d). Note also from comparing Figs. 1(b) and 1(d) that the water distribution at the polar tail is essentially unchanged (Table I). Returning to the solute-solute SDF of Fig. 1(c), interesting additional density appears (at between 8-8.5 $\AA$ ) in three lobes below the equator. Examination of the orientations of the $t$-butanol molecules in these lobes shows their polar groups point towards the polar groups of the central $t$-butanol molecule. Thus we conclude that these neighboring amphiphile molecules are associated in some way through the polar groups at the tail end of the amphiphile.

TABLE I. Selected coordination numbers for a 0.02 mole fraction aqueous solution of $t$-butanol, without and with added $\mathrm{NaCl}$. CC refers to the central carbon of the $t$-butanol molecule, $\mathrm{O}$ and $\mathrm{O}_{w}$ to the alcohol oxygen and water oxygen, respectively.

\begin{tabular}{ccccc}
\hline \hline Correlation & $\begin{array}{c}R_{\min } \\
(\AA)\end{array}$ & $\begin{array}{c}R_{\max } \\
(\AA)\end{array}$ & $\begin{array}{c}\text { Coord. No. } \\
\text { NaCl absent } \\
\text { (atoms) }\end{array}$ & $\begin{array}{c}\text { Coord. No. } \\
\text { NaCl present } \\
\text { (atoms) }\end{array}$ \\
\hline CC-CC & 4.3 & 7.5 & $1.5 \pm 0.7$ & $0.8 \pm 0.5$ \\
& 7.5 & 10.0 & $1.0 \pm 0.6$ & $1.8 \pm 0.8$ \\
$\mathrm{O}-\mathrm{O}$ & 2.5 & 3.5 & $0.02 \pm 0.08$ & $\cdots$ \\
$\mathrm{O}-\mathrm{O}_{w}$ & 2.5 & 3.5 & $2.5 \pm 0.6$ & $2.4 \pm 0.6$ \\
$\mathrm{Na}_{w}-\mathrm{O}_{w}$ & 2.1 & 3.0 & $\cdots$ & $4.2 \pm 0.1$ \\
$\mathrm{Cl}-\mathrm{O}_{w}$ & 2.8 & 3.6 & $\cdots$ & $4.9 \pm 0.1$ \\
\hline \hline
\end{tabular}




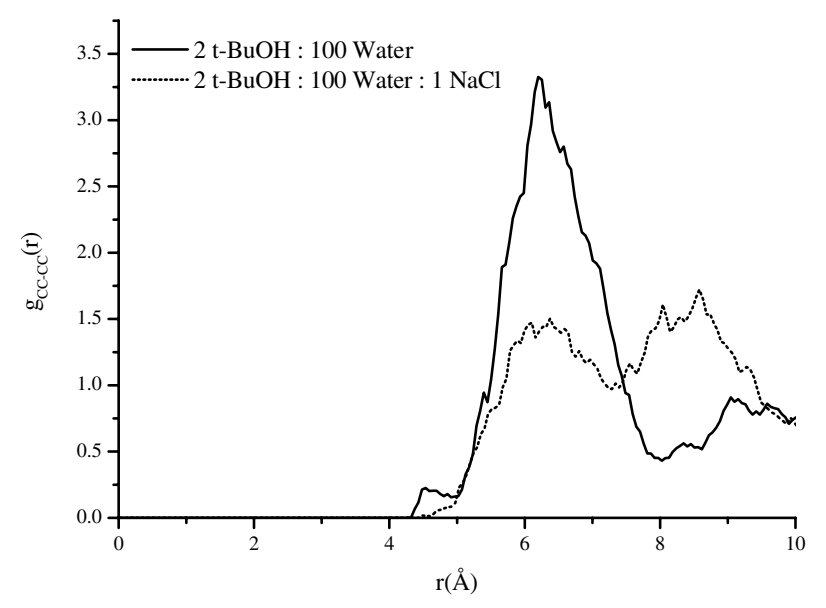

FIG. 2. Intermolecular partial radial distribution function $g_{c c-c c}(r)$ relating the central carbon atoms of neighboring $t$-butanol molecules. Solid line: no salt; dashed line: with added salt.

The distance of $8-8.5 \AA$ between the central carbon atoms of two neighboring $t$-butanol molecules is however too long for a direct contact between the alcohol molecules. Moreover, the O-O coordination numbers (Table I) and rdfs (not shown) show clearly that a direct hydrogen bonded interaction is not consistent with the experimental results. The nature of this intermolecular contact is made clear by referring to the SDF of the chloride ions around the alcohol shown in Fig. 1(e): we again see three lobes in the same direction as the $t$-butanol neighbors [Fig. 1(c)] but at a shorter distance. This indicates that the $t$-butanol association in this direction is mediated by a chloride ion. Calculations of possible CC-CC intermolecular distances involving a bridging chloride (around $8 \AA$ ) are totally consistent with the observed distance of $8-8.5 \AA$ of the second peak in the rdf of Fig. 2. We therefore conclude that the intermolecular $t$-butanol contact in this direction is through a direct anion salt bridge.

To complete the picture of the hydration structures in this solution we consider the hydration of the ions. Figure 3 (solid line) shows the partial ion-water oxygen rdf for sodium. Integration under the first peak gives a coordination number of essentially 4 (see Table I), a value which agrees with that obtained for sodium ions in an aqueous solution from a range of measurements and simulations [19-24]. The cation therefore retains its normal hydration shell in this solution. Its interaction with the amphiphile is shown in the TBA-Na ${ }^{+}$SDF of Fig. 1(f): like the "band" of water shown in Fig. 1(b), the sodium ions girdle the equator of the $t$-butanol. The ions are however farther out than the waters in this direction at a CC-Na ${ }^{+}$distance of approximately $7 \AA$, consistent with a picture in which some of the waters in the hydrating girdle are part of the cation hydration shell. Thus, the sodium ion keeps its hydration shell and links through this to the remaining nonpolar hydration shell of the

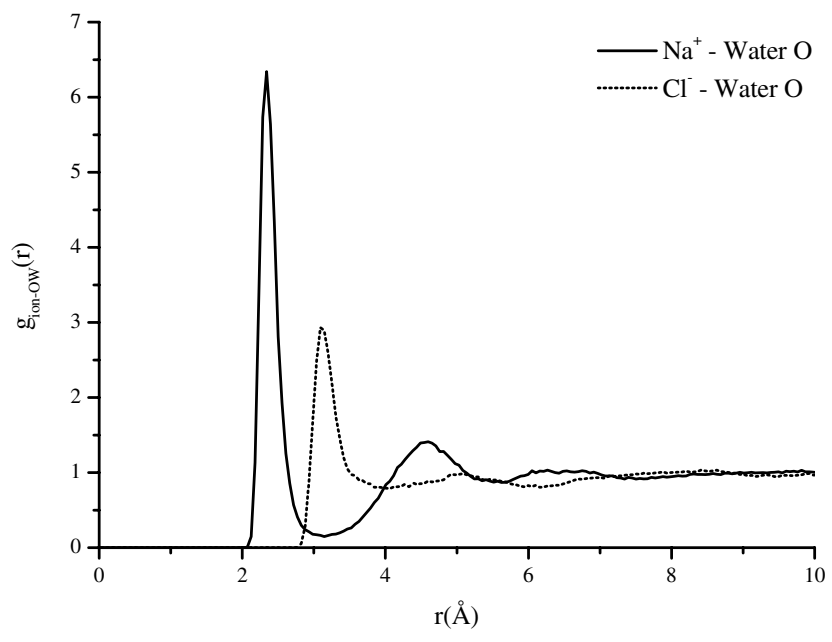

FIG. 3. Partial radial distribution functions showing the distribution of water oxygen atoms round the ions. Solid line: around sodium; dotted line: around chloride.

$t$-butanol. The structure of this water is found to be not significantly perturbed by the presence of the sodium. Turning now to the anion, Fig. 3 (dashed line) shows its hydration through the $\mathrm{Cl}$-water oxygen rdf. Integrating over the first sharp peak gives a coordination number of about 5 (see Table I). Comparing this with the expected value of 6 from a range of direct and indirect studies $[23,25]$ suggests that the anion has lost on average one of its nearest neighbor water molecules. This reduction is consistent with a picture in which about half the chloride ions bridge two alcohol molecules, while the other half remain fully hydrated in solution. If each of the bridging anions has two waters displaced by the two bridged alcohol $\mathrm{OH}$ groups, then this is consistent with the observed average chloride coordination of $\approx 5$.

To conclude, the effect of $\mathrm{NaCl}$ on an aqueous solution of this simple amphiphile is seen to be both dramatic and relatively simple. The cation remains fully hydrated, and although many sodium ions are in the proximity of the nonpolar region of the amphiphile, and link to the hydration water, they do not significantly perturb the nonpolar hydration shell. The anion effect is the key to the salt-induced association of the alcohol molecules. It draws away up to about one-half of the $t$-butanol molecules that were previously interacting through a hydrophobically driven nonpolar-nonpolar head-to-head contact towards a clearly polar association of the amphiphile's polar tails. In doing so, the anion loses two of its hydrating water molecules.

These results give us a vivid, and interestingly relatively simple, picture of what may be happening in the salting-out process. Rather than stress the importance of the salt in perturbing the hydration shell of the nonpolar part of the amphiphile, the process appears to be driven by polar interactions. This is not to say that as a whole the "hydrophobic" hydration of the nonpolar regions of the 
amphiphile is not relevant to the salting-out process. The molecular reorganization of the associated solute structures serves to expose more of the nonpolar surface to the bulk solvent water. Within the classical model of hydrophobic association, where molecular association is driven to minimize this exposure, the result of the addition of salt is thus to make the aggregated solutes less favorable to the aqueous environment.

These results further suggest that attempts to understand salting out through studies on purely nonpolar molecules are not appropriate - the amphiphilic nature of the molecule is crucial to the process itself, and therefore must be part of the system studied in order to understand it. Moreover, the apparent central importance of the salt bridge interaction may give us clues in the search for a rationale of the Hofmeister series which ranks ions in terms of their effectiveness in salting out amphiphiles. Two possible processes might be considered here: the need to partially dehydrate the anion and the ability of the partially stripped anion to fit between the polar groups of the amphiphile. The relative ease with which the various ions may participate in these processes may give us the key to this series.

We thank the Director of the ISIS Facility for the provision of the Director's Discretionary neutron beam time and support facilities.

[1] F. Hofmeister, Arch. Exp. Path. Pharmakol. 24, 247 (1888).

[2] H. R. Mahler and E. H. Cordes, Biological Chemistry (Harper and Row, New York, 1971).

[3] T. Arakawa and S. N. Timasheff, Biochemistry 21, 6545 (1982).

[4] H. Chick and C. J. Martin, Biochem. J. 7, 380 (1913).

[5] M. Holz, R. Grunder, A. Sacco, and A. Meleleo, J. Chem. Soc. Faraday Trans. 89, 1215 (1993).
[6] A. Sacco, F. M. De Cillis, and M. Holz, J. Chem. Soc. Faraday Trans. 94, 2089 (1998).

[7] D.W. Urry, Angew. Chem. Int. Ed. Engl. 32, 819 (1993).

[8] D. T. Bowron, J. L. Finney, and A. K. Soper, J. Phys. Chem. B 102, 3551 (1998).

[9] D. T. Bowron, A. K. Soper, and J. L. Finney, J. Chem. Phys. 114, 6203 (2001).

[10] J. Turner, A. K. Soper, and J. L. Finney, Mol. Phys. 77, 411 (1992).

[11] S. Dixit, J. Crain, W. C. K. Poon, A. K. Soper, and J. L. Finney, Nature (London) 416, 829 (2002).

[12] D. A. Armitage, M. J. Blandamer, K.W. Morcom, and N. C. Treloar, Nature (London) 219, 718 (1968).

[13] M. Mayerle, M. Holz, and A. Sacco, Phys. Chem. Chem. Phys. 1, 4615 (1999).

[14] A. K. Soper, W. S. Howells, and A. C. Hannon, Report No. RAL-89-046, 1989.

[15] K. Nakanishi, N. Kato, and M. Maruyama, J. Phys. Chem. 71, 814 (1967).

[16] A. K. Soper, Chem. Phys. 202, 295 (1996).

[17] A. K. Soper, Mol. Phys. 99, 1503 (2001).

[18] J. L. Finney and D. T. Bowron, in From Semiconductors to Proteins: Beyond the Average Structure, edited by S. F. Billinge and M. F. Thorpe (Kluwer Academic/Plenum, New York, 2002), pp. 219-244.

[19] T. Osakai, A. Ogata, and K. Ebina, J. Phys. Chem. B 101, 8341 (1997).

[20] K. Nortmann, J. Hilland, and U. Kaatze, J. Phys. Chem. A 101, 6864 (1997).

[21] R. Buchner, G. T. Hefter, and P. M. May, J. Phys. Chem. A 103, 1 (1999).

[22] A. Zavitsas, J. Phys. Chem. B 105, 7805 (2001).

[23] A. Zasetsky and I. M. Svishchev, J. Chem. Phys. 115, 1448 (2001).

[24] S. B. Rempe and L. R. Pratt, Fluid Phase Equilib. 183, 121 (2001).

[25] J. E. Enderby and G.W. Neilson, in Water. A Comprehensive Treatise, edited by F. Franks (Plenum Press, New York, 1979), Vol. 6, pp. 1-46. 